OPEN ACCESS

Edited by:

Grigorios Nasios,

University of loannina, Greece

Reviewed by:

Nicholas J. Kelley,

University of Southampton,

United Kingdom

Ya Zheng,

Dalian Medical University, China

${ }^{*}$ Correspondence:

Feng Zou

zoufeng@xxmu.edu.cn

Meng Zhang

mengzhang.1985@163.com

${ }^{t}$ These authors have contributed equally to this work

Specialty section:

This article was submitted to Neuropsychology,

a section of the journal

Frontiers in Psychology

Received: 18 November 2021

Accepted: 21 January 2022

Published: 18 February 2022

Citation:

Zou F, Li X, Chen F, Wang Y,

Wang $L$, Wang $Y$, Wu $X$ and Zhang $M$

(2022) P2 Manifests Subjective

Evaluation of Reward Processing

Under Social Comparison.

Front. Psychol. 13:817529.

doi: 10.3389/fpsyg.2022.817529

\section{P2 Manifests Subjective Evaluation of Reward Processing Under Social Comparison}

\author{
Feng Zou ${ }^{1 \star t}$, Xiaoya $\mathrm{Li}^{1+}$, Fenfang Chen ${ }^{2}$, Yao Wang ${ }^{1}$, Li Wang ${ }^{1}$, Yufeng Wang ${ }^{1}$, Xin Wu ${ }^{1}$ \\ and Meng Zhang ${ }^{1 *}$
}

${ }^{1}$ Department of Psychology, Xinxiang Medical University, Xinxiang, China, ${ }^{2}$ Department of Education, Hangzhou Normal University, Hangzhou, China

Several recent studies have found that when the other's gain is greater, even subjects' reward may seem like a loss and lead to a negative experience. These studies indicate the complexity of reward evaluation in the context of social comparison. The satisfaction rating of reward outcome not only depends on objective social comparison but also on subjective evaluation. However, less is known about the neural time course of subjective evaluation. Therefore, we employed a 2 (subjective evaluation: advantageous vs. disadvantageous) $\times 2$ (comparison direction: upward vs. downward) within-subjects factorial design, in which we manipulated the reward distribution for the subjects. Electroencephalography (EEG) responses were recorded, while two subjects concurrently but independently performed a simple dot-estimation task that entailed monetary rewards. Behavioral results showed that the subjects were more satisfied with the advantageous distribution, regardless of upward or downward comparison. The analysis of event-related potentials (ERPs) revealed that disadvantageous distribution elicited a larger P2 than advantageous distribution, and this effect was not modulated by comparison direction. In contrast, the late positive potential (LPP) showed an effect of comparison direction independent of subjective evaluation. The data suggest that subjective evaluation acts upon the early stage of reward processing and manifests in the P2 component, whereas social comparison plays a role in the later appraisal process.

Keywords: subjective evaluation, social comparison, reward evaluation, P2, LPP

\section{INTRODUCTION}

Social comparison is the process of thinking about the information of one or more other people in relation to the self (Wood, 1996). In daily life, social comparison is ubiquitous and plays a crucial role in human adaptation and survival. For example, people may make an upward comparison (compared with a superior other) hoping to improve their abilities (Collins, 1996) or make a downward comparison (compared with an inferior other) to increase self-evaluations (Wills, 1981). In recent years, many studies have concentrated on the influence of social comparison on reward processing. Social psychologists have shown that the subjective wellbeing of people not only depends on absolute income but also on relative income (Smith et al., 1989; Hagerty, 2000). Fliessbach et al. (2007) explored the impact of social comparisons on reward processing using 
functional MRI. They found that activity in the ventral striatum, a reward-related brain structure, is affected by the variation in the comparison of payments of the participants (Fliessbach et al., 2007). This finding was verified by other studies using slightly different paradigms, suggesting an immediate impact of social comparison on ventral striatal responses (Dvash et al., 2010; Bault et al., 2011; Dohmen et al., 2011). These studies from the perspectives of behavior and brain mechanisms highlight the importance of studying reward processing in the context of social comparison.

With the advantage of high time resolution of electroencephalography (EEG) technology, previous research has identified that social comparison may affect outcome event-related potential (ERP) components, including the $\mathrm{P} 2$, the feedback-related negativity (FRN), the P300, and the late positive potential (LPP) (Qiu et al., 2010; Wu et al., 2012; Kedia et al., 2014; Luo et al., 2015). The first P2 component is a positive, fronto-central ERP component, peaking around 150-250 ms post-feedback onset (Hillyard et al., 1973; Salim et al., 2015). A recent study suggested that the brain processes social comparison information at an early stage of outcome evaluation, with a larger P2 amplitude when subjects faced outcomes that were different from others (Luo et al., 2015). However, several other related studies did not find this component (Qiu et al., 2010; Wu et al., 2012). Consistent with the reward processing domain, the P2 component is thought to reflect attention selection and salience detection (Carretié et al., 2005; Potts et al., 2006; Bellebaum et al., 2010; San Martín et al., 2010; Xu et al., 2011; Schuermann et al., 2012; Flores et al., 2015; Glazer et al., 2018; Wischnewski and Schutter, 2018, 2019).

A second ERP component related to social comparison is the FRN, which is a fronto-central negative deflection, peaking around $250 \mathrm{~ms}$ post-feedback onset. Traditionally, the FRN is thought to code a reward prediction error in the human brain and reflects the evaluation of outcome along a goodbad dimension (Holroyd and Coles, 2002; Hajcak et al., 2007; Sambrook and Goslin, 2015). Several ERP studies tested whether social comparison modulated the FRN. For example, using a simple time-estimation reaction-time task, Boksem et al. (2011) found that enhanced FRN was elicited when the outcomes were worse than those of another player (Boksem et al., 2011). However, two studies employing similar experimental designs did not find FRN modulations related to social comparison (Qiu et al., 2010; Wu et al., 2012). These inconsistent results may be because the FRN is modulated by several factors, such as outcome probability, expectations, and perceived task difficulty, which vary with experimental paradigm changes (Kedia et al., 2014).

A third ERP component is the P300, which is a centroparietal positive-going deflection, peaking from 300 to $600 \mathrm{~ms}$ after feedback onset. Some studies have shown that the maximum period of the P300 component may be extended to approximately $800 \mathrm{~ms}$ (Bonnefond and der Henst, 2009; Li et al., 2014). Previously, the P300, which was frequently reported in the reward processing, was generally considered to be related to the motivationally significant or relevant stimuli that capture attentional resources (Yeung and Sanfey, 2004; Hajcak et al., 2005; Nieuwenhuis et al., 2005; Sato et al., 2005; Wu and Zhou, 2009; Zhou et al., 2010). So far, only one study found that the P300 component was modulated by the social comparison direction, not the feedback valence (Wu et al., 2012).

Finally, the LPP is a positive-going centro-parietal ERP component starting around $500 \mathrm{~ms}$ after feedback onset and lasts for several hundred milliseconds (Cuthbert et al., 2000; Schupp et al., 2006; Hajcak et al., 2009). In the context of reward processing, previous studies often conflate the P300 and LPP measurements because of their strong temporal overlap, but less is known about the functional differences between P3 and LPP in outcome processing (see review, Glazer et al., 2018). Whereas the LPP in the social comparison situations was often observed and modulated by comparison direction, the more unequally, the larger the LPP amplitude detailed (Qiu et al., 2010; Wu et al., 2011; Luo et al., 2015).

Previous research has shown that upward comparison elicits negative emotional responses and downward comparison elicits positive emotional reactions (Tesser and Collins, 1988; Aspinwall and Taylor, 1993; White et al., 2006; Dvash et al., 2010). However, in reality, individuals often encounter more than one comparison object, and the degree of difference between individual and comparison object is also diverse. In this study, Du and colleagues (2013) examined satisfaction with outcomes in a three-person comparison. In their experiment, one subject and two pseudo-subjects completed a simple number estimation task and received different monetary rewards. Interestingly, behavioral results suggested that despite the two reward distributions representing an upward comparison, the subjects were more satisfied with one distribution scheme (e.g., subject/pseudo-A/pseudo-B $=20 / 40 / 60$ ) than the other distribution scheme (e.g., subject/pseudo-A/pseudo$B=20 / 50 / 50)$. This finding shows that satisfaction rating seems to be influenced by subjective evaluation besides comparison direction. Specifically, in the upward comparison context, comparison objects with small differences may be evaluated as subjectively advantageous stimuli compared to comparison objects with large differences, which can improve individual satisfaction to some extent. Consistent with this point of view, another experiment found that even when subjects won money, they expressed envy if another player won more money. In contrast, when they lost money, they expressed joy and schadenfreude if another player lost more money (Dvash et al., 2010). Despite this phenomenon being more in line with actual life experiences of the people, the underlying neurobiological substrate of this social comparison process is not well understood.

With EEG recording, the primary objective of this study was to explore how subjective evaluation moderates the satisfaction rating of reward outcome in the context of social comparison and how the brain responds to such modulation. To this end, we revised the simple dot-estimation task used by Fliessbach et al. (2007). Two subjects concurrently but independently performed a simple dot-estimation task and received the monetary reward 
TABLE 1 | Reward conditions in the experiment.

\begin{tabular}{lccc}
\hline & Upward & Downward & Fillers \\
\hline Advantageous & $10-20$ & $40-10$ & $0-0$ \\
Disadvantageous & $10-40$ & $20-10$ & $0-10 / 10-0$ \\
$10-10 / 40-40$
\end{tabular}

There are four conditions of interest: advantageous upward (labeled "AU" below), advantageous downward ("AD"), disadvantageous upward ("DU"), and disadvantageous downward ("DD"). When both subjects completed the task correctly, one of four possible reward conditions ( $A U, A D, D U$, and $D D$ ) was randomly selected. For example, the two numbers ra0-20numbers randomly selected. For-upward comparison context, one subject received $10 ¥$, another player received $20 ¥$. Meanwhile, to establish a more realistic experiment, we set the filling conditions, in which at least one of the two subjects was incorrect, and both subjects were correct but received the same amount of money.

on each trial. The experiment employed a 2 (subjective evaluation: advantageous vs. disadvantageous) $\times 2$ (comparison direction: upward vs. downward) within-subject factorial design, in which we manipulated the reward distribution for the subjects. Reward conditions are illustrated in Table 1. Locke (1976) proposed that satisfaction is driven by the congruence between what we obtain and what we value (Locke, 1976). In this sense, people are relatively satisfied with these two kinds of reward distribution: they all get rewards, but they get more than others (i.e., self/others: $40 / 10$ vs. $20 / 10$ ), or although they get less than others, the difference is not too much (i.e., self/others: 10/20 vs. 10/40). Therefore, the two reward distributions are defined as subjectively advantageous conditions. In contrast, the other two reward distributions (i.e., self/others: 20/10 and 10/40) are defined as subjectively disadvantageous conditions. According to the previous neurophysiological studies, evaluation of reward outcome can be divided broadly into two related processes: first, an early evaluation of the motivational/affective significance of the reward feedback stimuli, which may manifest in the P2 or FRN component, followed by a more elaborative evaluation, which may manifest in the P300 or LPP (Goyer et al., 2008; Leng and Zhou, 2010; Wu et al., 2012). We anticipate that subjective evaluation as a factor would be processed at the abovementioned stages. However, due to the confounding of relevant research findings and the limited evidence for direct research on subjective evaluation, it is unlikely to make the formation of an accurate prediction model extremely.

\section{MATERIALS AND METHODS}

\section{Participants}

Forty-eight healthy students (24 women; mean age $18.48 \pm 0.90$ years) from Xinxiang Medical University participated in the experiment. Two subjects had to be excluded from the analysis due to excessive ocular artifacts, so that data from 46 subjects were finally analyzed. All subjects were righthanded and had normal or corrected-to-normal vision. None of them had a history of neurological or psychiatric problems. All subjects gave written informed consent in accordance with the Declaration of Helsinki. Furthermore, for subjects under 18 years of age, we obtained the written informed consent of their parents. The Institutional Ethics Committee of Xinxiang Medical University approved the study protocol.

\section{Procedure}

Each round of the experiment includes two subjects of the same gender to control the potential impact of gender differences in reward processing (Fliessbach et al., 2007; Warthen et al., 2020). Before the experiment, both subjects were asked that they would sit in two adjacent cabins to finish a simple number estimation task simultaneously through the computer network. They did not know each other and had no chance to communicate before the end of the experiment. They were also informed that the computer would allocate monetary rewards based on their performance in the task (i.e., response accuracy and response time). Unknown to the subjects, their performance (whether the estimates were right or wrong) and reward distribution were presented in a pseudo-randomized and counterbalanced way.

From the perspective of the subjects, reward feedback could be either upward comparison (when the subject got less money than the other player) or downward comparison (when the subject got more money than the other player). During upward comparison conditions, the reward feedback could be subjectively advantageous (reward distribution with a small difference, i.e., 10-20) or subjectively disadvantageous (reward distribution with a large difference, i.e., 10-40). In contrast, during downward comparison conditions, reward distribution with a small difference (i.e., 20-10) represents a subjectively disadvantageous condition, but reward distribution with a large difference (i.e., 40-10) represents a subjectively advantageous condition. There are four conditions of interest (refer to Table 1), namely, advantageous upward (labeled "AU” below), advantageous downward (“AD”), disadvantageous upward ("DU”), and disadvantageous downward ("DD"). When both subjects completed the task correctly, one of the four reward conditions (i.e., $\mathrm{AU}, \mathrm{AD}, \mathrm{DU}$, and $\mathrm{DD}$ ) was randomly selected. Meanwhile, to establish a more realistic experiment, we set the filling conditions, in which at least one of the two subjects was incorrect, and both subjects were correct but received the same amount of money.

Each trial began with a screen with a varying number of white dots (10-50) for 1,000 ms (refer to Figure 1). Subsequently, subjects had to judge the number of dots as "odd" or "even" by pressing the key " 1 " or " 2 ," respectively. The presentation stopped when subjects had responded. Then, a screen presented "Correct" or "Incorrect" for 1,000 ms as response feedback. The phrase "data matching" was presented for another 2,000 ms, implying that the computer was doing background processing in line with the performance of both subjects. Next, a reward feedback screen was displayed for 3,000 ms. This screen showed both players whether they were correct or not (indicated by a tick or a cross), together with the respective monetary rewards in this trial. Finally, subjects were required to rate their satisfaction with the reward feedback by pressing one of the numeric keys 1-5 (1 representing "very unsatisfied" and 5 representing "very satisfied"). The next trial started after a time interval of $250 \mathrm{~ms}$.

During the estimation task, the subject was seated comfortably about $1 \mathrm{~m}$ from a 19 -in CRT display. The experiment was 


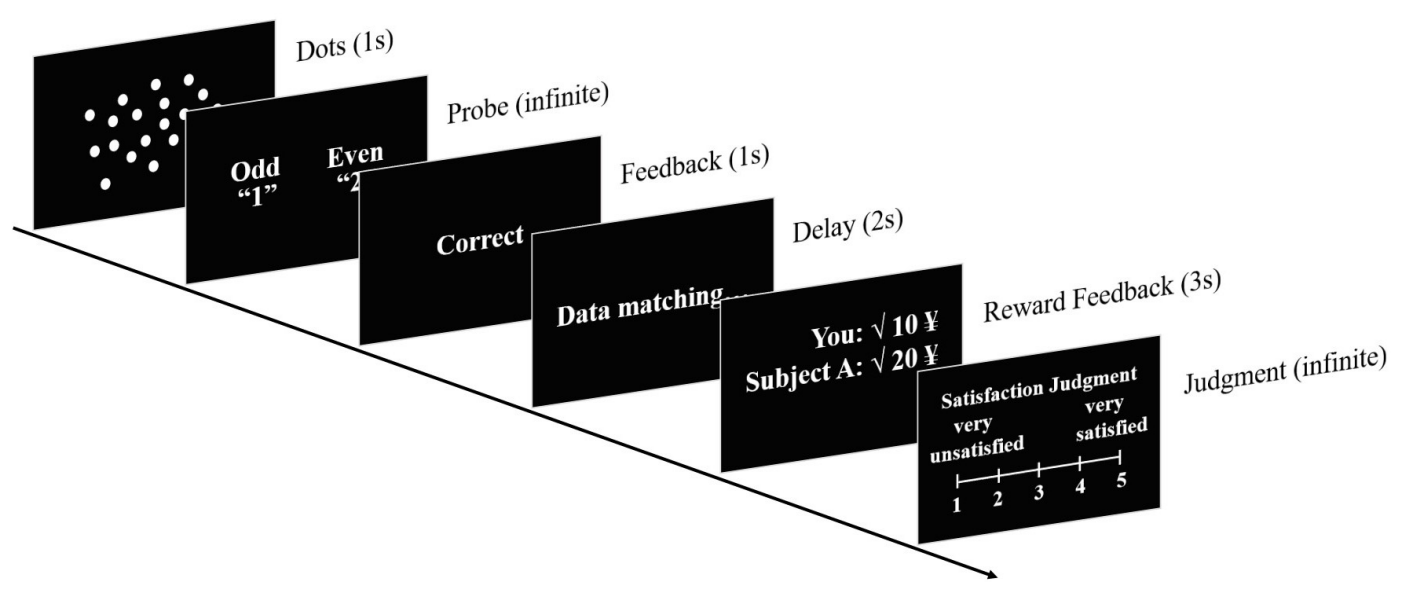

FIGURE 1 | A sequence of events in a single trial.

coded and performed using E-Prime 2.0 software (Psychology Software Tools, Pittsburgh, PA, United States). One of the four experimental conditions (AU/AD/DU/DD) had 35 trials. In addition, another 112 trials, corresponding to the filling conditions, were used as fillers. The 252 trials were randomly mixed and were divided in equal numbers into two blocks. Subjects could take a rest between the two blocks. At the end of the experiment, each subject was paid and thanked for their participation.

\section{Electroencephalography Recording and Analysis}

Continuous EEG was acquired using Neuroscan (Compumedics, Charlotte, United States) SynAmps2 64-channel amplifier and an $\mathrm{Ag} / \mathrm{AgCl}$ Electro-Cap according to the standard international 10-20 system. A cephalic (forehead) location was used for the ground, and the left mastoid was chosen for reference. Vertical electrooculogram (EOG) was recorded from electrodes placed above and below the left eye. Horizontal EOG was recorded from electrodes placed at the left and right orbital rim. All interelectrode impedance was kept below $5 \mathrm{k} \Omega$. The EEG signal was amplified with a band-pass filter from 0.01 to $100 \mathrm{~Hz}$ and digitized online at a sampling rate of $500 \mathrm{~Hz}$.

The EEGLAB toolbox (Makeig et al., 2004) under the MATLAB environment was used for offline analysis. Data were re-referenced to the mean of the left and right mastoids and were digitally filtered from 0.1 to $30 \mathrm{~Hz}$. Epochs were extracted from $0.2 \mathrm{~s}$ before to $1 \mathrm{~s}$ after the onset of the stimulus of interest. After baseline correction ( -0.2 to $0 \mathrm{~s}$ ), ocular artifacts were rejected by the independent component analysis. Trials with amplitudes exceeding $\pm 80 \mu \mathrm{V}$ at any electrode were excluded from further analysis.

The ERP components analyzed included P2, FRN, P300, and LPP. Based on previous studies (Gehring and Willoughby, 2002; Yeung and Sanfey, 2004; Wu et al., 2012; Luo et al., 2015; Pornpattananangkul and Nusslock, 2015; Kwak et al., 2019) and topographical maps (refer to Figures 2, 3), the
$\mathrm{FC} 1, \mathrm{FCz}, \mathrm{FC} 2, \mathrm{C} 1, \mathrm{Cz}$, and $\mathrm{C} 2$ electrodes were included in the calculations of the P2 component; for the FRN, one classical electrode, FCz, was included; for the P300, one classical electrode, $\mathrm{Pz}$, was included; for the LPP, Cz, Cp1, $\mathrm{Cpz}, \mathrm{Cp} 2, \mathrm{P} 1, \mathrm{Pz}, \mathrm{P} 2$, and $\mathrm{POz}$ electrodes were included. We chose 230-270, 320-400, 350-450, and 500-800 ms after the onset of reward stimulus for analyzing P2, FRN, P300, and LPP. These time windows were selected according to visual inspection of waveforms and the classical definitions for these components.

Analysis of variance was conducted with three withinsubject factors, namely, subjective evaluation (advantageous vs. disadvantageous), comparison direction (upward vs. downward), and electrode. Simple effect analysis was conducted when the interaction effect was significant. The GreenhouseGeisser correction was used in all statistical analyses whenever appropriate. The Bonferroni correction was applied for multiple comparisons.

\section{RESULTS}

\section{Behavioral Results}

A 2 (subjective evaluation: advantageous vs. disadvantageous) $\times 2$ (comparison direction: upward vs. downward) repeated-measures ANOVA revealed a significant main effect of comparison direction, $F(1,45)=46.22$, $p<0.001$, $\eta^{2}=0.507$. Pairwise comparison showed that subjects were more satisfied with downward comparison (mean $\pm S D, 3.80 \pm 0.82$ ) than with upward comparison $(2.69 \pm 1.11)$. The main effect of subjective evaluation was significant, $F(1,45)=47.61$, $p<0.001, \eta^{2}=0.514$. Pairwise comparison showed that a satisfaction rate for advantageous distribution $(3.39 \pm 1.11)$ was higher than that for disadvantageous distribution $(3.10 \pm 1.13)$. However, the comparison direction $\times$ subjective evaluation interaction effect was not statistically significant, $F(1,45)=0.94$, $p=0.338, \eta^{2}=0.020$. The behavioral result is presented in Figure 4. 


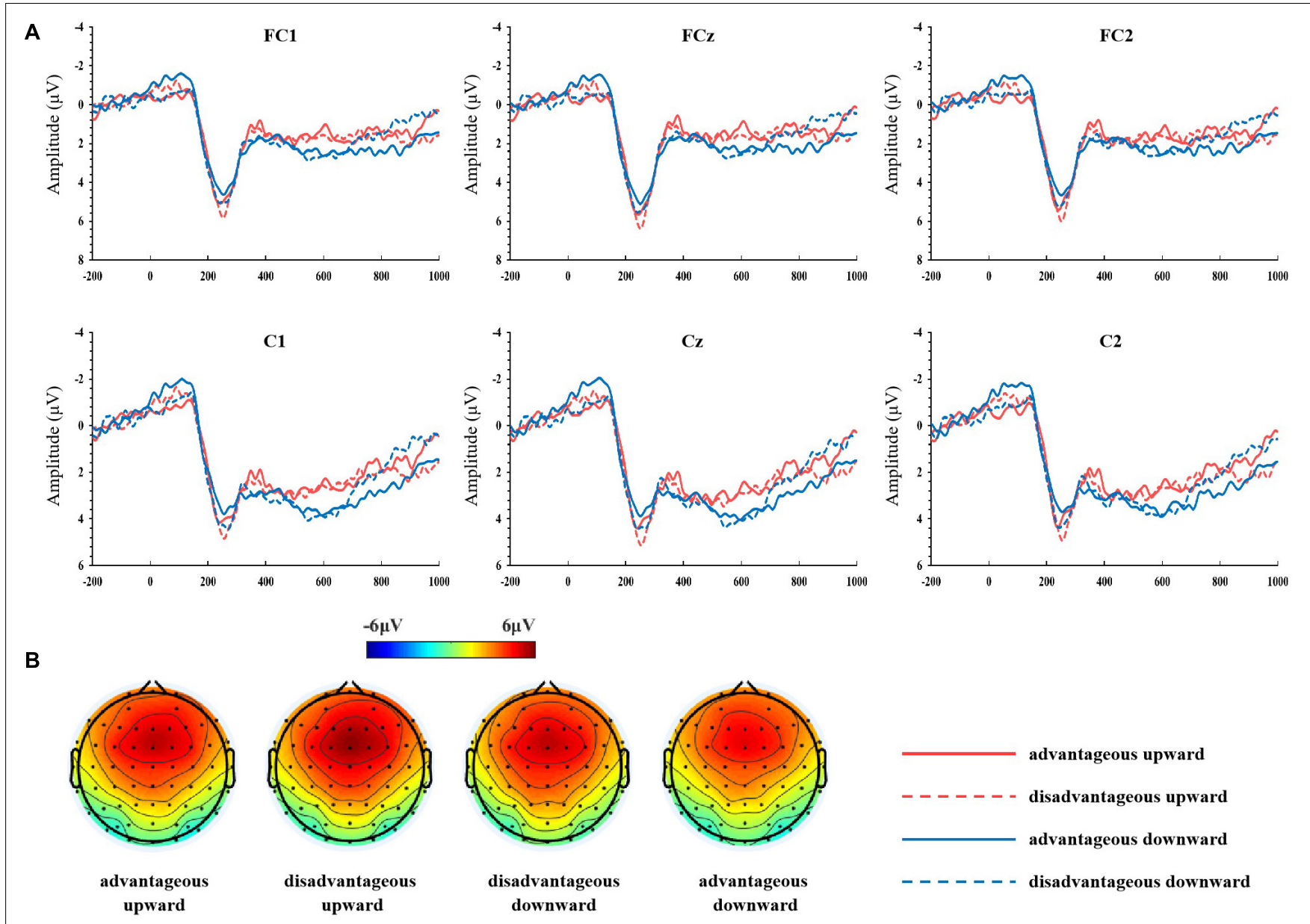

FIGURE 2 | (A) Grand-averaged event-related potentials (ERPs) at FC1, FCz, FC2, C1, Cz, and C2 electrodes for P200 with a comparison of the outcome under various feedback conditions. (B) Topographical voltage distributions of four conditions of interest in the time range of the P2 (230-270 ms).

\section{Event-Related Potential Results}

Analysis of the P2 component revealed a significant main effect for the subjective evaluation factor, $F(1,45)=4.17$, $p<0.05, \eta^{2}=0.085$. Pairwise comparison showed that the disadvantageous distribution (mean $\pm S D, 4.93 \pm 0.44$ $\mu \mathrm{V}$ ) elicited a larger P2 than the advantageous distribution $(4.36 \pm 0.41 \mu \mathrm{V})$. The main effect of comparison direction factor was not significant, $F(1,45)=1.59, p=0.214, \eta^{2}=0.034$. No significant interaction of comparison direction $\times$ subjective evaluation was found, $F(1,45)=0.24, p=0.625, \eta^{2}=0.005$ (refer to Figure 2A).

No significant effects were observed for the FRN component (subjective evaluation: $F(1,45)=0.001, p=0.977, \eta^{2}<0.001$; comparison direction: $F(1,45)=3.541, p=0.066, \eta^{2}=0.073$; subjective evaluation $\times$ comparison direction: $F(1,45)=0.588$, $p=0.447, \eta^{2}=0.013$ ), indicating that the FRN was similar across $\mathrm{AU}, \mathrm{AD}, \mathrm{DU}$, and $\mathrm{DD}$ comparison conditions.

No significant effects were observed for the P300 component (subjective evaluation: $F(1,45)=1.305, p=0.259, \eta^{2}=0.028$; comparison direction: $F(1,45)=1.12, p=0.296, \eta^{2}=0.024$; subjective evaluation $\times$ comparison direction: $F(1,45)=0.317$, $\left.p=0.576, \eta^{2}=0.007\right)$, indicating that the $\mathrm{P} 300$ was similar across $\mathrm{AU}, \mathrm{AD}, \mathrm{DU}$, and DD comparison conditions.

Analysis of the LPP component revealed a significant main effect for the comparison direction factor, $F(1,45)=23.27$, $p<0.001, \eta^{2}=0.341$. Pairwise comparison showed that the LPP was larger in response to downward comparison $(3.88 \pm 0.37$ $\mu \mathrm{V})$ than upward comparison $(2.97 \pm 0.30 \mu \mathrm{V})$. Neither the main effect of the subjective evaluation factor, $F(1,45)=0.10$, $p=0.750, \eta^{2}=0.002$, nor the interaction of comparison direction $\times$ subjective evaluation, $F(1,45)=1.59, p=0.214$, $\eta^{2}=0.034$, was significant (refer to Figure 3A).

\section{DISCUSSION}

This study investigated the influence of subjective evaluation and comparison direction on reward processing under social comparison contexts. Consistent with previous studies (Dvash et al., 2010; San Martín et al., 2010; Du et al., 2013), we found that the subjects were more satisfied with reward distribution when they received more than other players. Furthermore, our results 


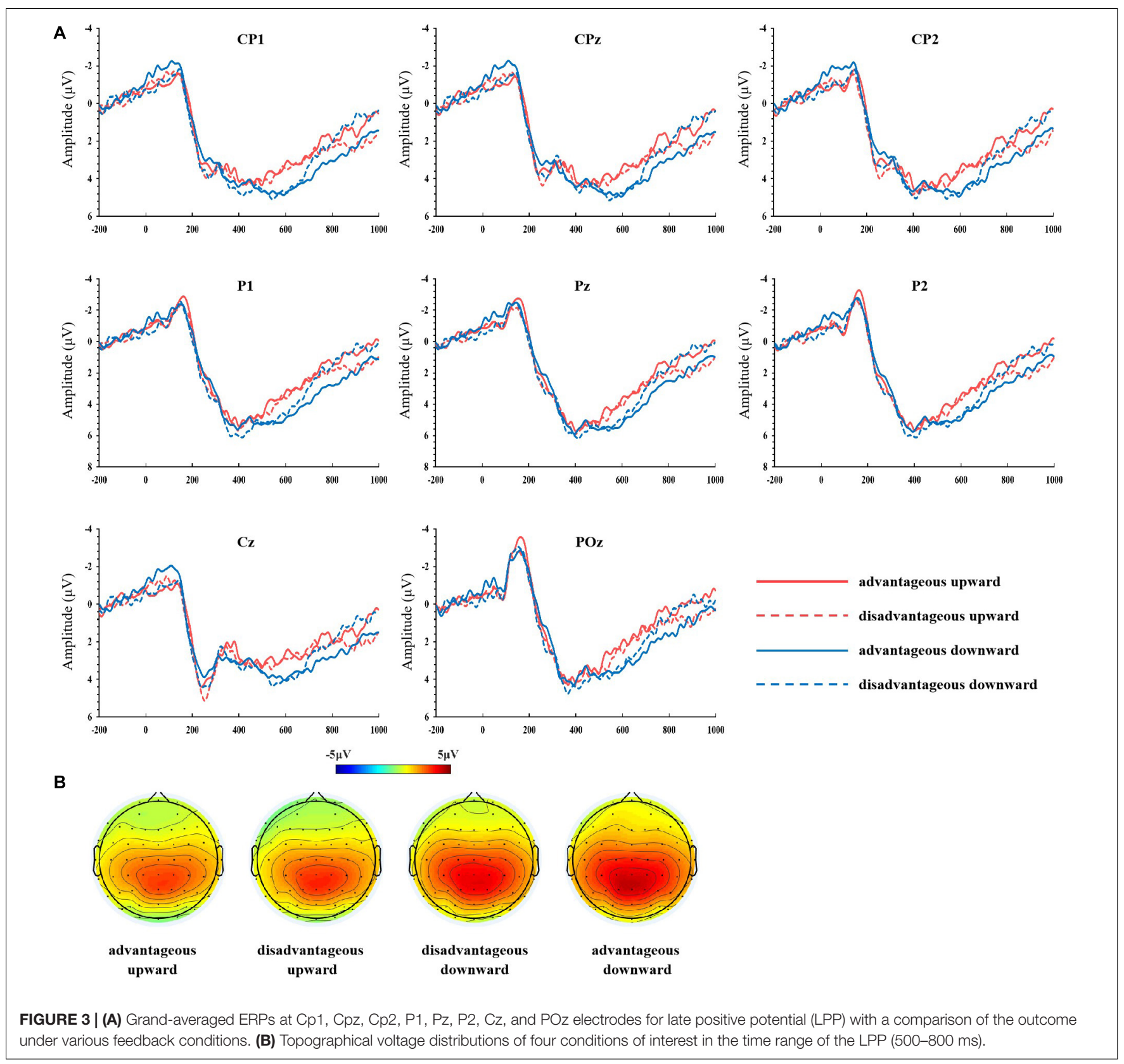

showed that the satisfaction rating was modulated by subjective evaluation. Specifically, the subjects were more satisfied with the advantageous distribution, regardless of upward or downward comparison. Electrophysiologically, we observed a subjective evaluation effect on the P2, and this effect was not modulated by comparison direction. In contrast, the LPP component displayed the modulation effect of comparison direction but was not sensitive to the subjective evaluation factor. However, the FRN and P300 components were neither sensitive to subjective evaluation nor comparison direction.

The early P2 component displayed a differential processing of advantageous and disadvantageous outcomes. Results show that the P2 amplitude was larger in response to disadvantageous distribution as compared to advantageous distribution. This finding suggests that the brain differentially processes advantageous and disadvantageous outcomes already at an early stage. Previous studies on the effects of valence have yielded rather inconclusive, and even conflicting, conclusions (Carretié et al., 2005; Bellebaum et al., 2010; Xu et al., 2011; Schuermann et al., 2012). Our findings support the view that the P2 is an arousal component that reflects a valence effect associated with feedback (Wischnewski and Schutter, 2018). It should be noted that most previous studies did not consider subjective evaluation as a variable factor. Indeed, the variable subjective evaluation represents the subjective judgment of an individual on the value of feedback outcome, which is not exactly the same 


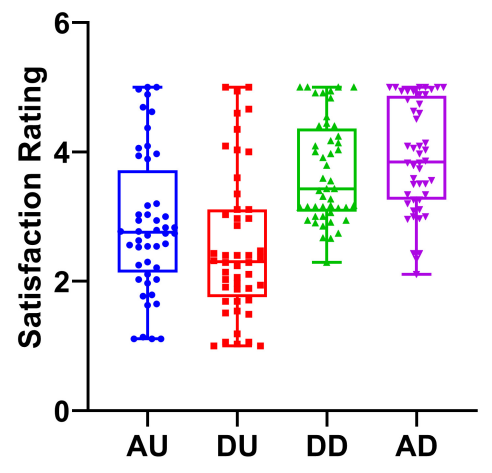

FIGURE 4 | Satisfaction rating under various feedback conditions. Advantageous upward condition is shown in blue; disadvantageous upward condition is shown in red; disadvantageous downward condition is shown in green; and advantageous downward condition is shown in purple.

as the valence concept involved in other studies (e.g., win/loss and positive/negative). Therefore, it may not be appropriate to directly compare the results of our experiment with those of other relevant studies. In this study, the modulation effect of P2 by subjective evaluation suggests that P2 may serve as a neural index for the binary classification of the advantageous and disadvantageous outcome. The P2 component plays a role in salience detection in the early stage of reward processing; in this sense, more attention was given to disadvantageous feedback.

The P2 amplitude was not modulated by comparison direction, a result inconsistent with those observed in a recent study on outcome evaluation (Luo et al., 2015). Their experiment employed a three-person comparison paradigm, in which researchers asked the subjects to play a lottery game with two pseudo-players simultaneously, and presented them with their outcome and those of two other players. The comparison between self and others showed hierarchical characteristics. They supposed that at the early stage of outcome evaluation, the processing of social comparison information was relatively rough, and the detailed information was not yet processed until later stages.

Unlike the P2, we found that the LPP component was modulated by social comparison. The LPP amplitude was larger when the subject received a monetary reward more than another player. The results are generally consistent with previous studies on outcome evaluation (Qiu et al., 2010; Wu et al., 2011, 2012). Traditionally, the LPP is thought to reflect sustained attention and extended cognitive processing of the motivationally salient, high-arousal stimuli (Schupp et al., 2000; Groen et al., 2008; Dunning and Hajcak, 2009; Althaus et al., 2010; Pornpattananangkul and Nusslock, 2015). In this study, reward feedback presented only one valence category, "gain," and the difference between the conditions was whether the subject received a monetary reward more or less than the other player. From a motivational perspective, the downward comparison conditions (e.g., self/others: 20/10 and 40/10) are evaluated as most motivational relevant in comparison with the upward comparison conditions (e.g., self/others: $10 / 20$ and 10/40).
Thus, we interpret this LPP effect as reflecting motivationally salient outcomes.

Moreover, our results did not find the FRN effect and P300 effect, which is inconsistent with many previous studies (Hajcak et al., 2005, 2007; Bellebaum et al., 2010; Kreussel et al., 2012). According to the independent coding model proposed by Yeung and Sanfey (2004), two key reward features, namely, valence (i.e., loss vs. gains) and magnitude (i.e., large vs. small), are encoded separately in the brain, with the FRN being sensitive to reward valence and the P3 to reward magnitude. However, other studies have shown that FRN and P300 are all affected by outcome probability, with unexpected outcomes eliciting larger amplitude (Campbell et al., 1979; Horst et al., 1980; Gibson et al., 2006; Hajcak et al., 2007; Goyer et al., 2008; Jessup et al., 2010; Alexander and Brown, 2011; Kreussel et al., 2012; San Martín, 2012; Talmi et al., 2013; Watts et al., 2017). Consistent with this point of view, in this study, the conditions of interest that had equal trials were presented in a pseudorandom manner. Therefore, subjects were not able to form a reliable reward prediction for a particular parity judgment and thus could not detect prediction errors. This may explain why FRN and P300 were not affected by subjective evaluation and comparison direction. However, it should be noted that the main effect of the comparison direction in this study was marginally significant. Therefore, researchers should be very cautious when interpreting the results.

\section{CONCLUSION AND LIMITATIONS}

To sum up, this study tentatively distinguishes subjective evaluation from objective social comparison. Behavioral results reveal the complexity of social comparison in the process of reward evaluation, that is, the satisfaction rating of reward outcome not only depends on social comparison but also on subjective evaluation. EEG results suggest that subjective evaluation acted upon earlier processing stage and manifested in the P2 component. This finding highlights the importance of P2 in reward processing and further extends the significance of the early ERP components of reward processing. Furthermore, in terms of theory, the strong temporal overlap among reward-related components (Glazer et al., 2018) and the complexity of social comparison itself lead to substantial conflict in the existing literature. Independent coding theory proposes that the two attributes of reward stimulus, i.e., valence and magnitude, are processed independently in the early and late stages of reward outcome processing, which are reflected in FRN and P300, respectively. However, this study showed that subjective evaluation also affected reward processing, as reflected in the $\mathrm{P} 2$, whereas the effect of comparison direction on reward processing was mainly in the later stage of outcome evaluation, as reflected in the LPP. This extends the theory to some extent. In the future, more research is needed to clarify how humans subjectively process feedback outcomes and what role these classical components play in this process. Moreover, given the complexity of social comparison and the flexibility of individuals, it is 
necessary to explain individual differences in future studies of social comparison.

\section{DATA AVAILABILITY STATEMENT}

The original contributions presented in the study are included in the article/supplementary material, further inquiries can be directed to the corresponding author/s.

\section{ETHICS STATEMENT}

The studies involving human participants were reviewed and approved by the Institutional Ethics Committee of Xinxiang Medical University. Written informed consent to participate in this study was provided by the participants' legal guardian/next of kin.

\section{AUTHOR CONTRIBUTIONS}

FZ contributed to the conceptualization, methodology, formal analysis, investigation, data curation, writing-original draft, writing-review and editing, and visualization. XL contributed

\section{REFERENCES}

Alexander, W. H., and Brown, J. W. (2011). Medial prefrontal cortex as an action-outcome predictor. Nat. Neurosci. 14, 1338-1344. doi: 10.1038/nn. 2921

Althaus, M., Groen, Y., Wijers, A. A., Minderaa, R. B., Kema, I. P., Dijck, J. D., et al. (2010). Variants of the SLC6A3 (DAT1) polymorphism affect performance monitoring-related cortical evoked potentials that are associated with ADHD. Biol. Psychol. 85, 19-32. doi: 10.1016/j.biopsycho.2010. 04.007

Aspinwall, L. G., and Taylor, S. E. (1993). Effects of social comparison direction, threat, and self-esteem on affect, self-evaluation, and expected success. J. Pers. Soc. Psychol. 64, 708-722. doi: 10.1037//0022-3514.64. 5.708

Bault, N., Joffily, M., Rustichini, A., and Coricelli, G. (2011). Medial prefrontal cortex and striatum mediate the influence of social comparison on the decision process. Proc. Natl. Acad. Sci. 108, 16044-16049. doi: 10.1073/pnas.110089 2108

Bellebaum, C., Kobza, S., Thiele, S., and Daum, I. (2010). It was not my fault: event-related brain potentials in active and observational learning from feedback. Cereb. Cortex 20, 2874-2883. doi: 10.1093/cercor/bh q038

Boksem, M. A. S., Kostermans, E., and De Cremer, D. (2011). Failing where others have succeeded: medial frontal negativity tracks failure in a social context. Psychophysiology 48, 973-979. doi: 10.1111/j.1469-8986.2010.01 163.x

Bonnefond, M., and der Henst, V. (2009). What's behind an inference? An EEG study with conditional arguments. Neuropsychologia 47, 3125-3133. doi: 10. 1016/j.neuropsychologia.2009.07.014

Campbell, K. B., Courchesne, E., Picton, T. W., and Squires, K. C. (1979). Evoked potential correlates of human information processing. Biol. Psychol. 8, 45-68. doi: 10.1016/0301-0511(79)90004-8

Carretié, L., Hinojosa, J. A., Mercado, F., and Tapia, M. (2005). Cortical response to subjectively unconscious danger. Neuroimage 24, 615-623. doi: 10.1016/j. neuroimage.2004.09.009

Collins, R. L. (1996). For better or worse: the impact of upward social comparison on self-evaluations. Psychol. Bull. 119, 51-69. to the investigation, formal analysis, data curation, and writingreview and editing. FC, YaW, LW, and YfW contributed to the investigation and writing-review and editing. MZ contributed to the conceptualization, methodology, formal analysis, writingoriginal draft, writing-review and editing, visualization, and supervision. All authors contributed to the article and approved the submitted version.

\section{FUNDING}

This research was supported by the National Natural Science Foundation of China (31600927 and 81830040), the Humanities and Social Science Research Project of Henan Colleges and Universities (2021-ZZJH-308), and the Youth Foundation of Social Science and Humanity, China Ministry of Education (21YJC190023 and 19YJCZH179).

\section{ACKNOWLEDGMENTS}

We would like to thank reviewers for their helpful comments. We thank LetPub (www.letpub.com) for its linguistic assistance during the preparation of this manuscript.

Cuthbert, B. N., Schupp, H. T., Bradley, M. M., Birbaumer, N., and Lang, P. J. (2000). Brain potentials in affective picture processing: covariation with autonomic arousal and affective report. Biol. Psychol. 52, 95-111. doi: 10.1016/ s0301-0511(99)00044-7

Dohmen, T., Falk, A., Fliessbach, K., Sunde, U., and Weber, B. (2011). Relative versus absolute income, joy of winning, and gender: brain imaging evidence. J. Public Econ. 95, 279-285.

Du, X., Zhang, M., Wei, D., Li, W., Zhang, Q., and Qiu, J. (2013). The Neural circuitry of reward processing in complex social comparison: evidence from an event-related fMRI study. PLoS One 8:e82534. doi: 10.1371/journal.pone. 0082534

Dunning, J. P., and Hajcak, G. (2009). See no evil: directing visual attention within unpleasant images modulates the electrocortical response. Psychophysiology 46, 28-33. doi: 10.1111/j.1469-8986.2008.00723.x

Dvash, J., Gilam, G., Ben-Ze'ev, A., Hendler, T., and Shamay-Tsoory, S. G. (2010). The envious brain: the neural basis of social comparison. Hum. Brain Mapp. 31, 1741-1750. doi: 10.1002/hbm.20972

Fliessbach, K., Weber, B., Trautner, P., Dohmen, T., Sunde, U., Elger, C. E., et al. (2007). Social comparison affects reward-related brain activity in the human ventral striatum. Science 318, 1305-1308. doi: 10.1126/science.114 5876

Flores, A., Münte, T. F., and Doñamayor, N. (2015). Event-related EEG responses to anticipation and delivery of monetary and social reward. Biol. Psychol. 109, 10-19. doi: 10.1016/j.biopsycho.2015.04.005

Gehring, W. J., and Willoughby, A. R. (2002). The medial frontal cortex and the rapid processing of monetary gains and losses. Science 295, 2279-2282. doi: $10.1126 /$ science. 1066893

Gibson, J., Krigolson, O. E., and Holroyd, C. B. (2006). Sensitivity of the feedback error-related negativity to reward probability. Psychophysiology 43, S41-S42.

Glazer, J. E., Kelley, N. J., Pornpattananangkul, N., Mittal, V. A., and Nusslock, R. (2018). Beyond the FRN: broadening the time-course of EEG and ERP components implicated in reward processing. Int. J. Psychophysiol. 132, 184202. doi: 10.1016/j.ijpsycho.2018.02.002

Goyer, J. P., Woldorff, M. G., and Huettel, S. A. (2008). Rapid electrophysiological brain responses are influenced by both valence and magnitude of monetary rewards. J. Neurosci. 20, 2058-2069. doi: 10.1162/jocn.2008. 20134 
Groen, Y., Wijers, A. A., Mulder, L. J., Waggeveld, B., Minderaa, R. B., and Althaus, M. (2008). Error and feedback processing in children with ADHD and children with autistic spectrum disorder: an EEG event-related potential study. Clin. Neurophysiol. 119, 2476-2493. doi: 10.1016/j.clinph.2008. 08.004

Hagerty, M. R. (2000). Social comparisons of income in one's community: evidence from national surveys of income and happiness. J. Pers. Soc. Psychol. 78, 764-771. doi: 10.1037//0022-3514.78.4.764

Hajcak, G., Dunning, J. P., and Foti, D. (2009). Motivated and controlled attention to emotion: time-course of the late positive potential. Clin. Neurophysiol. 120, 505-510. doi: 10.1016/j.clinph.2008.11.028

Hajcak, G., Holroyd, C. B., Moser, J. S., and Simons, R. F. (2005). Brain potentials associated with expected and unexpected good and bad outcomes. Psychophysiology 42, 161-170. doi: 10.1111/j.1469-8986.2005. 00278.x

Hajcak, G., Moser, J. S., Holroyd, C. B., and Simons, R. F. (2007). It's worse than you thought: the feedback negativity and violations of reward prediction in gambling tasks. Psychophysiology 44, 905-912. doi: 10.1111/j.1469-8986.2007. 00567.x

Hillyard, S. A., Hink, R. F., Schwent, V. L., and Picton, T. W. (1973). Electrical signs of selective attention in the human brain. Science 182, 177-180. doi: $10.1126 /$ science.182.4108.177

Holroyd, C. B., and Coles, M. G. H. (2002). The neural basis of human error processing: reinforcement learning, dopamine, and the error-related negativity. Psychol. Rev. 109, 679-709.

Horst, R. L., Johnson, R. Jr., and Donchin, E. (1980). Event-related brain potentials and subjective probability in a learning task. Mem. Cognit. 8, 476-488. doi: 10.3758/bf03211144

Jessup, R. K., Busemeyer, J. R., and Brown, J. W. (2010). Error effects in anterior cingulate cortex reverse when error likelihood is high. J. Neurosci. 30, 34673472. doi: 10.1523/JNEUROSCI.4130-09.2010

Kedia, G., Mussweiler, T., and Linden, D. E. (2014). Brain mechanisms of social comparison and their influence on the reward system. Neuroreport 25, 12551265. doi: 10.1097/WNR.0000000000000255

Kreussel, L., Hewig, J., Kretschmer, N., Hecht, H., Coles, M. G. H., and Miltner, W. H. R. (2012). The influence of the magnitude, probability, and valence of potential wins and losses on the amplitude of feedback negativity. Psychophysiology 49, 207-219. doi: 10.1111/j.1469-8986.2011.01 291.X

Kwak, Y., Chen, X., McDonald, K., and Boutin, B. (2019). Money for me and money for friend: an ERP study of social reward processing in adolescents and adults. Soc. Neurosci. 15, 83-97. doi: 10.1080/17470919.2019.165 3963

Leng, Y., and Zhou, X. (2010). Modulation of the brain activity in outcome evaluation by interpersonal relationship: an ERP study. Neuropsychologia 48, 448-455. doi: 10.1016/j.neuropsychologia.2009.10.002

Li, B., Zhang, M., Luo, J., Qiu, J., and Liu, Y. (2014). The difference in spatiotemporal dynamics between modus ponens and modus tollens in the wason selection task: an event-related potential study. Neuroscience 270, 177182. doi: 10.1016/j.neuroscience.2014.04.007

Locke, E. A. (1976). "The nature and causes of job satisfaction," in Handbook of industrial and organizational psychology, Vol. 1, ed. M. D. Dunnette (Chicago: Rand McNally), 1297-1349.

Luo, Y., Feng, C., Wu, T., Broster, L. S., Cai, H., Gu, R., et al. (2015). Social comparison manifests in event-related potentials. Sci. Rep. 5:12127. doi: 10. 1038/srep 12127

Makeig, S., Debener, S., Onton, J., and Delorme, A. (2004). Mining eventrelated brain dynamics. Trends Cogn. Sci. 8, 204-210. doi: 10.1016/j.tics.2004. 03.008

Nieuwenhuis, S., Slagter, H. A., Von Geusau, N. J., Heslenfeld, D. J., and Holroyd, C. B. (2005). Knowing good from bad: differential activation of human cortical areas by positive and negative outcomes. Eur. J. Neurosci. 21, 3161-3168. doi: 10.1111/j.1460-9568.2005.04152.x

Pornpattananangkul, N., and Nusslock, R. (2015). Motivated to win: relationship between anticipatory and outcome reward-related neural activity. Brain Cogn. 100, 21-40. doi: 10.1016/j.bandc.2015.09.002

Potts, G. F., Martin, L. E., Burton, P., and Montague, P. R. (2006). When things are better or worse than expected: the medial frontal cortex and the allocation of processing resources. J. Cogn. Neurosci. 18, 1112-1119. doi: 10.1162/jocn.2006. 18.7.1112

Qiu, J., Yu, C., Li, H., Jou, J., Tu, S., Wang, T., et al. (2010). The impact of social comparison on the neural substrates of reward processing: an event-related potential study. Neuroimage 49, 956-962. doi: 10.1016/j.neuroimage.2009.08. 025

Salim, M. A. M., van der Veen, F. M., van Dongen, J. D. M., and Franken, I. H. A. (2015). Brain activity elicited by reward and reward omission in individuals with psychopathic traits: an ERP study. Biol. Psychol. 110, 50-58. doi: 10.1016/ j.biopsycho.2015.07.001

Sambrook, T. D., and Goslin, J. (2015). A neural reward prediction error revealed by a meta-analysis of ERPs using great grand averages. Psychol. Bull. 141, 213-235. doi: 10.1037/bul0000006

San Martín, R. (2012). Event-related potential studies of outcome processing and feedback-guided learning. Front. Hum. Neurosci. 6:304. doi: 10.3389/fnhum. 2012.00304

San Martín, R., Manes, F., Hurtado, E., Isla, P., and Ibañez, A. (2010). Size and probability of rewards modulate the feedback error-related negativity associated with wins but not losses in a monetarily rewarded gambling task. Neuroimage 51, 1194-1204. doi: 10.1016/j.neuroimage.2010.03.031

Sato, A., Yasuda, A., Ohira, H., Miyawaki, K., Nishikawa, M., Kumano, H., et al. (2005). Effects of value and reward magnitude on feedback negativity and P300. Neuroreport 16, 407-411. doi: 10.1097/00001756-20050315000020

Schuermann, B., Endrass, T., and Kathmann, N. (2012). Neural correlates of feedback processing in decision-making under risk. Front. Hum. Neurosci. 6:204. doi: 10.3389/fnhum.2012.00204

Schupp, H. T., Cuthbert, B. N., Bradley, M. M., Cacioppo, J. T., Ito, T., and Lang, P. J. (2000). Affective picture processing: the late positive potential is modulated by motivational relevance. Psychophysiology 37, 257-261.

Schupp, H. T., Flaisch, T., Stockburger, J., and Junghöfer, M. (2006). Emotion and attention: event-related brain potential studies. Prog. Brain Res. 156, 31-51. doi: 10.1016/S0079-6123(06)56002-9

Smith, R. H., Diener, E., and Wedell, D. H. (1989). Intrapersonal and social comparison determinants of happiness: a range-frequency analysis. J. Pers. Soc. Psychol. 56, 317-325. doi: 10.1037//0022-3514.56. 3.317

Talmi, D., Atkinson, R., and El-Deredy, W. (2013). The feedback-related negativity signals salience prediction errors not reward prediction errors. J. Neurosci. 33, 8264-8269. doi: 10.1523/JNEUROSCI.5695-12.2013

Tesser, A., and Collins, J. E. (1988). Emotion in social reflection and comparison situations: intuitive, systematic, and exploratory approaches. J. Pers. Soc. Psychol. 55, 695-709. doi: 10.1037//0022-3514.55.5.695

Warthen, K. G., Boyse-Peacor, A., Jones, K. G., Sanford, B., Love, T. M., and Mickey, B. J. (2020). Sex differences in the human reward system: convergent behavioral, autonomic and neural evidence. Soc. Cogn. Affect. Neurosci. 15, 789-801.

Watts, A. T., Bachman, M. D., and Bernat, E. M. (2017). Expectancy effects in feedback processing are explained primarily by time-frequency delta not theta. Biol. Psychol. 129, 242-252. doi: 10.1016/j.biopsycho.2017.08.054

White, J. B., Langer, E. J., Yariv, L., and Welch, J. C. (2006). Frequent social comparisons and destructive emotions and behaviors: the dark side of social comparisons. J. Adult Dev. 13, 36-44. doi: 10.1007/s10804-0069005-0

Wills, T. A. (1981). Downward comparison principles in social psychology. Psychol. Bull. 90, 245-271. doi: 10.1037/0033-2909.90.2.245

Wischnewski, M., and Schutter, D. J. L. G. (2018). Dissociating absolute and relative reward- and punishment-related electrocortical processing: an event-related potential study. Int. J. Psychophysiol. 126, 13-19. doi: 10.1016/j.ijpsycho.2018. 02.010

Wischnewski, M., and Schutter, D. J. L. G. (2019). Electrophysiological correlates of prediction formation in anticipation of reward- and punishment-related feedback signals. Psychophysiology 56:e13379. doi: 10.1111/psyp.13379

Wood, J. V. (1996). What is social comparison and how should we study it? Pers. Soc. Psychol. Bull. 22, 520-537.

$\mathrm{Wu}, \mathrm{Y}$., and Zhou, X. (2009). The P300 and reward valence, magnitude, and expectancy in outcome evaluation. Brain Res. 1286, 114-122. doi: 10.1016/j. brainres.2009.06.032 
Wu, Y., Zhang, D., Elieson, B., and Zhou, X. (2012). Brain potentials in outcome evaluation: when social comparison takes effect. Int. J. Psychophysiol. 85, 145152. doi: 10.1016/j.ijpsycho.2012.06.004

Wu, Y., Zhou, Y., Dijk, E. V., Leliveld, M. C., and Zhou, X. (2011). Social comparison affects brain responses to fairness in asset division: an erp study with the ultimatum game. Front. Hum. Neurosci. 5:131. doi: 10.3389/fnhum. 2011.00131

Xu, Q., Shen, Q., Chen, P., Ma, Q., Sun, D., and Pan, Y. (2011). How an uncertain cue modulates subsequent monetary outcome evaluation: an ERP study. Neurosci. Lett. 505, 200-204. doi: 10.1016/j.neulet.2011. 10.024

Yeung, N., and Sanfey, A. G. (2004). Independent coding of reward magnitude and valence in the human brain. J. Neurosci. 24, 6258-6264. doi: 10.1523/ JNEUROSCI.4537-03.2004

Zhou, Z., Yu, R., and Zhou, X. (2010). To do or not to do? Action enlarges the FRN and P300 effects in outcome evaluation. Neuropsychologia 48, 3606-3613. doi: 10.1016/j.neuropsychologia.2010.08.010
Conflict of Interest: The authors declare that the research was conducted in the absence of any commercial or financial relationships that could be construed as a potential conflict of interest.

Publisher's Note: All claims expressed in this article are solely those of the authors and do not necessarily represent those of their affiliated organizations, or those of the publisher, the editors and the reviewers. Any product that may be evaluated in this article, or claim that may be made by its manufacturer, is not guaranteed or endorsed by the publisher.

Copyright (c) 2022 Zou, Li, Chen, Wang, Wang, Wang, Wu and Zhang. This is an open-access article distributed under the terms of the Creative Commons Attribution License (CC BY). The use, distribution or reproduction in other forums is permitted, provided the original author(s) and the copyright owner(s) are credited and that the original publication in this journal is cited, in accordance with accepted academic practice. No use, distribution or reproduction is permitted which does not comply with these terms. 